\title{
DIÁLOGOS INTERSETORIAIS: PONTES ESTABELECIDAS NA REVISÃO DA POLÍTICA NACIONAL DE PROMOÇÃO DA SAÚDE
}

\section{INTERSECTORAL DIALOGUES: BRIDGES ESTABLISHED BY THE REVISION OF THE NATIONAL POLICY FOR HEALTH PROMOTION IN BRAZIL}

\author{
Roberto Eduardo Bueno ${ }^{1}$ \\ Simone Tetu Moysés ${ }^{2}$ \\ Paula Alexandra Reis Bueno ${ }^{3}$
}

\begin{abstract}
Resumo
Objetivou-se explorar a intersetorialidade como princípio, diretriz e eixo operacional da Política Nacional de Promoção da Saúde (PNPS) a partir de sua implantação e síntese das percepções e proposições de especialistas vinculados a instituições fora do Setor Saúde e movimentos sociais envolvidos em ações intersetoriais de promoção da saúde que apoiaram a revisão da PNPS em 20132014. Optou-se pela técnica Delphi, com a participação de representantes da Secretaria Geral da Presidência da República, Ministérios das Cidades e da Educação, Secretarias de Estado, Prefeituras, Organizações Não Governamentais, Fundações, Serviço Social da Indústria e Universidades. Do total de 32 respondentes, 90,6\% afirmaram conhecer a PNPS no momento da pesquisa. O grupo de respondentes reafirmou a relação estruturante da promoção da saúde com o desenvolvimento sustentável e o protagonismo social, reforçando a necessidade de atuação sobre os determinantes sociais da saúde, identificados como os condicionantes étnico-raciais e de gênero, as condições de vida no trabalho, o planejamento urbano e rural, a mobilidade e acessibilidade, além da violência. A contribuição de especialistas oportunizou o fortalecimento de uma nova versão da PNPS estabelecendo pontes para o diálogo intersetorial e suporte ao desenvolvimento destas ações no contexto brasileiro.
\end{abstract}

Palavras-chave: Ação Intersetorial; Saúde Coletiva; Formulação de Políticas; Desenvolvimento Sustentável; Determinantes Sociais da Saúde; Políticas Públicas.

\begin{abstract}
This study aimed to explore the intersectionality as a principle, policy and operational axis of the National Health Promotion Policy (PNPS) in Brazil from its implementation and synthesis of perceptions and proposals of experts linked to institutions outside the health sector and social movements involved in intersectoral actions health promotion that supported the review of PNPS in 2013-2014. We opted for the Delphi technique, with the participation of representatives of the Presidency General Secretariat of State, Ministry of Cities and Education, Secretaries of State, municipalities, NGOs, Foundations, Social Service for Industry and Universities. Of the 32 respondents, $90.6 \%$ said they knew the PNPS at the time of the survey. The group of respondents reaffirmed the structural relationship of health promotion with sustainable development and social leadership, reinforcing the need for action on the social determinants of health identified as ethnic and racial conditions and gender, living conditions at work, urban and rural planning, mobility and
\end{abstract}

\footnotetext{
Dossiê: Recebido em 30/05/2017 - Aprovado em 23/08/2017

${ }^{1}$ Pós-doutorado em Saúde Coletiva, Câmara do Curso de Saúde Coletiva, Setor Litoral, Universidade Federal do Paraná. $e$ mail: roberto.edu.bueno@gmail.com (autor correspondente)

2 Doutora em Epidemiologia e Saúde Pública, Escola de Saúde e Biociências, Pontifícia Universidade Católica do Paraná, Filiação.e-mail: simone.moyses@pucpr.br

${ }^{3}$ Doutora em Educação, Faculdade de Educação, Universidade de São Paulo.e-mail: paula.reis@ usp.br
} 
accessibility, as well as violence. The contribution of experts provided an opportunity to strengthen a new version of PNPS building bridges for inter-sectoral dialogue and support the development of these actions in the brazilian context.

Keywords: Intersectoral Action; Public Health; Policy Making; Sustainable Development; Social Determinants of Health; Public Policies.

\section{Introdução}

A intersetorialidade é apresentada na Política Nacional de Promoção da Saúde (PNPS) em três dimensões estabelecidas como pontes: princípios, diretrizes e eixos operacionais. Estas pontes estão interconectadas para os diálogos imprescindíveis no desenvolvimento das ações intersetoriais. A intersetorialidade como princípio, configura-se em todas as práticas no campo da promoção da saúde. Como diretriz, explicita a sua finalidade como linha de atuação sobre os determinantes e condicionantes da saúde. E como eixo operacional, representa uma estratégia de concretizar ações de promoção da saúde por meio de articulação e cooperação intersetorial, ou seja, pelo compartilhamento de planos, metas, recursos e objetivos comuns entre os diferentes setores (BRASIL, 2015).

A PNPS de 2015 revisa os conteúdos formulados na sua primeira versão em 2006 e ratifica a intersetorialidade como princípio, ao referi-la como processo de articulação de saberes, potencialidades e experiências de sujeitos, grupos e setores na construção de intervenções compartilhadas, estabelecendo vínculos, corresponsabilidade e cogestão para objetivos comuns. Entre os objetivos específicos da PNPS, encontra-se o de contribuir para a articulação de políticas públicas intersetoriais com as agendas nacionais e internacionais. A PNPS também indica temas prioritários para as ações intersetoriais de promoção da saúde compatíveis com o Plano Nacional de Saúde em permanente diálogo com as políticas de saúde e de outros setores e com as especificidades sanitárias regionais. A PNPS está ancorada no conceito ampliado de saúde. Ela sinaliza estratégias para a promoção da qualidade de vida e para a redução de vulnerabilidade e riscos à saúde relacionados aos seus determinantes e condicionantes sociais, tais como: modos de vida, condições de trabalho, habitação, ambiente, educação, lazer, cultura, acesso a bens e serviços essenciais, sendo que para isso é fundamental a atuação intersetorial para minimizar as iniquidades sociais em saúde (BRASIL, 2006; BRASIL, 2015).

A Declaração de Adelaide e a Declaração de Helsínquia sobre a Saúde em Todas as Políticas identificaram a ação intersetorial e as políticas públicas saudáveis como elementos primordiais para a promoção da saúde e para a realização da equidade em saúde. Houve convergência que a Saúde é meta fundamental para os governos que visam o desenvolvimento sustentável. A saúde é um direito humano fundamental e a equidade em saúde expressa a justiça social. A saúde melhora a qualidade de vida, capacidade de aprendizado, produtividade no trabalho e empodera as famílias e comunidades. Políticas elaboradas envolvendo todos os setores aprofundam o impacto sobre a equidade em saúde de uma população. A abordagem da "Saúde em Todas as Políticas" integra as políticas públicas em todos os setores, promovendo a participação ampla em decisões para a saúde e buscando potencializar as ações intersetoriais de promoção da saúde, com o objetivo de melhorar a saúde e a equidade social da população (OMS, 2010; THE HELSINKI STATEMENT ON HEALTH IN ALL POLICIES, 2013).

O enfoque "Saúde em Todas as Políticas" tem como característica uma base sólida de direitos humanos e justiça social com ênfase na formulação de políticas, cuja aplicação envolve a identificação de oportunidades para o desenvolvimento de políticas em todos os setores com implicações em potencial para a saúde e a equidade em saúde. Isto se dá pela concordância entre a promoção da saúde, os determinantes sociais da saúde e os direitos humanos. Uma das grandes contribuições deste enfoque é o seu potencial para combater o crescimento das doenças não transmissíveis. Isto reforçará a integração de práticas nos diversos sistemas, tanto na saúde como nos outros setores, ampliando o alcance e a sustentabilidade das políticas e a responsabilidade pela saúde por todos os setores (OMS, 2014) 
A política pública de saúde brasileira, regulamentada nas Leis 8080 e 8142 em 1990, expressa no Sistema Único de Saúde (SUS), tem na sua concepção diversos fundamentos convergentes com as Conferências Mundiais de Promoção da Saúde, entre eles a descentralização e a participação social, que fomentam a articulação de ações intersetoriais sobre os determinantes sociais da saúde. No SUS, a promoção da saúde é retomada como uma estratégia de ampliar a visão sobre tudo o que determina o processo saúde-doença-cuidado como, por exemplo: violência, desemprego, saneamento básico, habitação, acesso à educação, fome, urbanização, qualidade do ar e da água; e potencializar modos intersetoriais e equitativos de intervenção em saúde (BRASIL, 1990a; BRASIL, 1990b).

O planejamento e a articulação intersetorial devem atuar sobre a interconexão entre saúde e desenvolvimento humano como está detalhado na publicação da Comissão Internacional de Determinantes Sociais de Saúde da Organização Mundial da Saúde, que entende a saúde como um recurso para o desenvolvimento econômico sustentável e enfatiza que as condições de vida também são capazes de determinar a saúde de uma população. Esta constatação fica explícita na relação entre uma maior intensidade de pobreza e mais altos níveis de doença e mortalidade prematura. Essas iniquidades em saúde são evitáveis e injustas socialmente e estão acometendo os seres humanos mais vulneráveis (COMMISSION ON SOCIAL DETERMINANTS OF HEALTH, 2007).

Uma pesquisa embasou-se em um Modelo Conceitual para Desenvolvimento Humano e Promoção da Saúde, que partiu da hipótese de que sustentabilidade, governança e equidade possuem interfaces em comum. A partir desse pressuposto, o estudo examinou quais seriam as inter-relações teórico-conceituais entre sustentabilidade, governança e equidade, sob a perspectiva da relação desenvolvimento-saúde. Foram originadas três interfaces: políticas de governo e estado, balanço de poder e processo inclusivo e resultados impactantes direcionados à promoção da saúde. Em sua maioria, foram significativamente contempladas na VIII Conferência Local e nas propostas inseridas no Plano Municipal de Saúde 2010-2013 de São José dos Pinhais (BUENO et al., 2013).
A construção de uma agenda de pesquisa, que reforce o referencial teórico para uma governança interesetorial, é imprescindível para que as ações intersetoriais representem a práxis de governo sustentável e informada por evidências científicas na resolução de problemas complexos e comuns (AKERMAN et al., 2014).

Em uma pesquisa qualitativa, com seis municípios da região metropolitana de Belo Horizonte, foi revelada a intersetorialidade como premissa na definição política da maior parte das práticas. O setor de assistência social demonstrou maior potencial para desenvolver práticas intersetoriais e centralidade na rede de articulação com os determinantes socioambientais. Houve um distanciamento entre a intenção de praticar a intersetorialidade e a sua efetivação no cotidiano. Concluiu-se que há potencialidade para intervenções intersetoriais sobre os determinantes socioambientais, mas há inconsistências entre $\mathrm{O}$ praticado e os aspectos políticos. À medida que houver mobilização de outros campos de saber e prática profissional no sentido prático da intersetorialidade, amplia-se a capacidade de atuação sobre os determinantes socioambientais de promoção da saúde (SILVA et al., 2014).

Em outro estudo foi analisada a PNPS quanto à implementação de eixos prioritários de gestão. Houve avanços na gestão como a criação de linha orçamentária específica, a inserção da Promoção da Saúde no Plano Plurianual, no monitoramento de indicadores nos pactos federativos, no financiamento de projetos em municípios e na criação de programas. Ações intersetoriais implementadas foram relevantes, em especial a articulação com os setores de Educação, Justiça, Cidades, Direitos Humanos, Desenvolvimento Social, Esporte e Lazer, dentre outros. Ações regulatórias foram implementadas, como a Lei "Seca" e a lei de ambientes livres de tabaco. Observaram-se avanços e fortalecimento da Promoção da Saúde nas ações intersetoriais, na identificação das iniquidades no território, e na sustentabilidade das práticas (MALTA et al., 2014).

Pesquisadores perceberam em um estudo que a realização de práticas intersetoriais é ainda um desafio, mas que é uma prática possível, principalmente com suporte e estímulo da gestão local. O conceito e a prática da intersetorialidade 
precisam ser inseridos na rotina de gestores e profissionais de saúde. As respostas e discursos das equipes e gestores locais demonstraram que é possível mudar e melhorar as realidades locais (MORETTI et al., 2010).

O objetivo deste artigo foi explorar a discussão da intersetorialidade como princípio, diretriz e eixo operacional da PNPS e resgatar as oportunidades e a implementação de ações intersetoriais apontadas pela PNPS no Brasil a partir de sua implantação e síntese das percepções e proposições de especialistas vinculados a instituições fora do Setor Saúde e movimentos sociais envolvidos em ações intersetoriais de promoção da saúde que apoiaram a revisão da PNPS conduzida em 2013-2014.

\section{Método}

Como forma de ampliar a participação e garantir a diversidade de perspectivas no processo de revisão da PNPS, este estudo se propôs a identificar a percepção de especialistas vinculados a instituições fora do setor saúde e movimentos sociais envolvidos em ações intersetoriais de promoção da saúde, construindo consensos e dissensos, e sintetizando as contribuições do grupo para a construção/reconstrução de objetivos, diretrizes, estratégias e ações específicas, inovadoras e socialmente inclusivas no âmbito das ações de Promoção da Saúde.

A abordagem metodológica utilizada no processo de revisão da PNPS está pautada na participação dos diferentes atores e setores envolvidos na construção e implementação de práticas de promoção da saúde no Brasil. Isto se deu pela aproximação intersetorial com informantes chave de outros Ministérios e Secretarias do Governo, bem como de instituições não governamentais selecionadas, por meio da utilização do método Delphi.

O Delphi é uma técnica para a busca de um consenso de opiniões, idéias e estratégias para a proposição de políticas por um grupo de especialistas, a partir de um questionário interativo online, que circula repetidas vezes entre o grupo, preservando o anonimato das respostas individuais. A técnica baseia-se no uso estruturado do conhecimento, da experiência e da criatividade de experts no tema analisado, pressupondo-se que o julgamento coletivo é melhor que a opinião de um só indivíduo (YAÑEZ; CUADRA, 2008).

Foram identificados e convidados a participar desta pesquisa 145 especialistas vinculados às instituições fora do setor saúde e movimentos sociais envolvidos em ações intersetoriais de promoção da saúde. Tais especialistas foram selecionados com base em informações, fornecidas por: a) Ministério da Saúde/ Coordenação Geral de Doenças e Agravos Não Transmissíveis, sobre pessoas de referência envolvidas em ações intersetoriais já desenvolvidas em parceria com outros setores, instituições, ministérios e movimentos sociais; b) consulta as listas de participantes de eventos nacionais ligados ao tema da intersetorialidade na promoção da saúde (ex.: Encontro Nacional da Rede de Municípios/ Territórios Saudáveis e Sustentáveis; encontros temáticos sobre a Agenda 21 brasileira); c) contatos com os membros do Grupo Temático Pró-Rede em Promoção da Saúde e Desenvolvimento Local Integrado e Sustentável da Associação Brasileira de Saúde Coletiva (ABRASCO); d) um último grupo foi identificado a partir de sua participação em reunião de secretariado sobre a revisão da PNPS realizada em Brasília no final do mês de abril de 2014, composto por representantes de Ministérios e da Casa Civil da Presidência da República.

Após a construção da relação dos nomes dos especialistas selecionados nos meses de janeiro e fevereiro, os emails de contato foram individualmente confirmados. Um convite para participar do processo de consulta sobre o eixo II do Plano Nacional de Enfrentamento das Doenças Crônicas subsidiando a revisão da Política Nacional de Promoção da Saúde, informando sobre a realização da pesquisa, seus objetivos e metodologia a ser utilizada, foi então enviado via email para um primeiro grupo de respondentes no dia 25 de fevereiro de 2014. A cada grupo convidado era dado um prazo médio de 10 dias para a resposta. No convite era enviado o link de acesso ao instrumento a ser respondido em plataforma web. A primeira rodada de consultas foi concluída em 14 de março de 2014.

O método Delphi, caracterizado como um método sistemático e interativo que busca consenso de opiniões com base na experiência independente de cada especialista foi utilizado para consulta aos especialistas convidados sobre concepções, 
princípios e valores da promoção da saúde, além dos objetivos, diretrizes, temas, estratégias e eixos operacionais da PNPS (YANEZ; CUADRA, 2008; PIOLA; VIANNA; VIVAS-CONSUELO, 2002).

Após a concordância com o Termo de Consentimento Livre Esclarecido, os participantes foram convidados a responder um questionário interativo via web. A primeira rodada de consultas ocorreu nos meses de fevereiro e março de 2014. Participaram desta rodada 32 especialistas.

O material obtido nesta primeira rodada foi analisado por meio da análise de distribuição de frequências das respostas objetivas, considerando consenso do grupo quando houve concordância igual ou maior que $70 \%$. Nova versão do questionário, com um sumário das estimativas efetuadas na primeira rodada, contendo apenas as questões onde houve dissenso, foi então enviada aos participantes para uma segunda rodada de consultas. Os especialistas foram encorajados a revisar suas respostas anteriores com base nas opiniões dos demais participantes. A segunda rodada de consultas ocorreu nos meses de abril e maio de 2014. Participaram deste momento da pesquisa 12 especialistas.

Os resultados finais obtidos das respostas quantitativas foram expressos pela distribuição de frequências totais e relativas. As questões abertas foram analisadas por meio da análise de conteúdo proposta por Bardin, que incluiu inicialmente uma leitura flutuante do material obtido e posteriormente a busca dos consensos e dissensos (BARDIN, 2009).

Uma síntese das respostas quantitativas e qualitativas foi então construída, consolidando a opinião do grupo sobre o tema.

As contribuições do grupo para o processo de revisão da PNPS ligado ao eixo II do Plano Nacional de Enfrentamento das Doenças Crônicas foram apresentadas em Oficinas de Consenso e no Seminário Nacional de revisão da PNPS em Brasília, no mês de maio de 2014 .

\section{Resultados e discussão}

A idade média dos respondentes foi de 47,6 anos (mínima 26, máxima 89). Do total, 14 (56,2\%) eram do sexo feminino e $18(43,7 \%)$ do sexo masculino.
A formação profissional dos respondentes incluiu as áreas de Arquitetura e Urbanismo, Medicina, Psicologia, Letras, Economia, Fisioterapia, Enfermagem, Direito, Musicoterapia, Sociologia, Serviço Social, Educação Física, Engenharia Civil, Fonoaudiologia e Pedagogia.

Do total, 27 respondentes $(84,3 \%)$ relataram ter PósGraduação e $5(15,7 \%)$ deles relataram formação em Graduação.

Participaram como respondentes pessoas vinculadas à Secretaria Geral da Presidência da República, Ministério das Cidades, Ministério da Educação, Secretarias de Estado, Prefeituras, Administração Regionais, Instituto de Planejamento Urbano, Universidades e centros de pesquisa, além de representantes do Serviço Social da Indústria, Fundação para o Desenvolvimento Sustentável e ONGs. O tempo médio de atuação nestas instituições foi de 12 anos, variando de menos de 1 ano a 30 anos.

Foram relatadas as participações em estratégias e práticas de promoção da saúde ligadas ao desenvolvimento local saudável e sustentável, meio ambiente e soluções urbanas, saúde, humanização, integração de políticas sociais, participação e inclusão, práticas integrativas em saúde, saúde do trabalhador, educação, além de ações específicas de combate à violência e estímulo a comportamentos saudáveis.

Do total de respondentes, 90,6\% afirmaram conhecer a PNPS no momento da pesquisa, conforme Tabela 1.

Tabela 1 - Resultados da pergunta: Você conhece a Política Nacional de Promoção da Saúde (PNPS)?

\begin{tabular}{lcc}
\hline $\begin{array}{l}\text { Conhecimento } \\
\text { da PNPS }\end{array}$ & Contagem & Porcentagem \\
\hline Sim & & \\
Não & 29 & 90,6 \\
\hline Total & 3 & 9,4 \\
\hline Fonte: Os Autores (2017) & & 100 \\
\hline
\end{tabular}

Após a análise das respostas às perguntas objetivas e subjetivas, uma síntese da percepção do grupo de participantes é apresentada a seguir, considerando as manifestações sobre concepções e conceitos de promoção da saúde; princípios e valores; consensos, dissensos e sugestões de inclusão ligados aos objetivos, diretrizes, temas prioritários, estratégias e eixos operacionais da 
PNPS; além de recomendações para a construção da nova versão da PNPS.

A análise de conteúdo revelou as dimensões que serão agora sequencialmente apresentadas.

\subsection{Concepções e conceitos}

O grupo de respondentes reafirmou a relação estruturante da promoção da saúde com o desenvolvimento sustentável e o protagonismo social, reforçando a necessidade de atuação sobre os determinantes sociais da saúde, identificados como os condicionantes étnico-raciais e de gênero, as condições de vida no trabalho, o planejamento urbano e rural, a mobilidade e acessibilidade, além da violência.

A integralidade foi considerada como valor importante da promoção da saúde, devendo ser construída a partir de uma clara diferenciação entre ações de promoção da saúde e a atenção à saúde, assumida exclusivamente pelo setor saúde.

\subsection{Princípios e valores priorizados}

De acordo com o grupo de respondentes, os princípios e valores mais importantes a serem considerados na PNPS foram a capacitação profissional, qualificação dos trabalhadores e educação permanente; o controle social/participação social; a intersetorialidade; o empoderamento; a sustentabilidade das ações; além da avaliação e monitoramento de ações e a efetividade dos serviços.

\subsection{Objetivos da PNPS}

Para a maioria dos respondentes, o objetivo geral e os objetivos específicos da atual PNPS respondiam em parte às demandas atuais de promoção da saúde e se aproximavam do perfil de ações de promoção da saúde com as quais estavam envolvidos em seu setor de atuação.

Houve consenso do grupo em relação à essencial manutenção dos objetivos específicos ligados à ampliação da autonomia e da corresponsabilidade; favorecimento da preservação do meio ambiente e a promoção de ambientes mais seguros e saudáveis; contribuição para elaboração e implementação de políticas públicas integradas; bem como valorização e aumento da cooperação do Setor Saúde com outras áreas de governo. O consenso do grupo em relação a estes objetivos certamente manifesta a coerência com a importância dada a ação integrada, intersetorial e participativa na construção de práticas de promoção da saúde.

Apesar de terem sido considerados importantes, não houve consenso sobre a essencialidade dos objetivos específicos ligados à implementação de ações de promoção da saúde com ênfase na atenção básica e a contribuição para o aumento da resolubilidade do sistema (de saúde), estes focados na perspectiva de responder às demandas setoriais, bem como os objetivos ligados ao estímulo a alternativas inovadoras e socialmente inclusivas, e a processos de integração baseados na solidariedade e gestão democrática, talvez por terem sido considerados mais como diretrizes, que como objetivos da Política.

O grupo propôs a inclusão de outros objetivos no novo texto da PNPS que incluem: formação multiprofissional em promoção da saúde; empoderamento local com reforço a modos de produção solidários e sustentáveis; atuar sobre dimensões da qualidade de vida, como a relação entre espiritualidade e saúde; monitoramento e avaliação de impacto sobre indicadores de qualidade de vida; valorizar o saber popular.

\subsection{Diretrizes da PNPS}

As respostas do grupo de especialistas evidenciaram consenso sobre as diretrizes ligadas ao reconhecimento da promoção da saúde como parte fundamental da busca da equidade, da melhoria da qualidade de vida e saúde; o estímulo às ações intersetoriais; o fortalecimento da participação social; a construção de redes de cooperação apoiadas pela gestão participativa; e a importância da produção de conhecimentos sobre a efetividade das práticas.

Entretanto, houve dissenso no grupo quanto a essencialidade da permanência da diretriz ligada a divulgação e informação de iniciativas voltadas para a promoção da saúde para profissionais de saúde, 
gestores e usuários do SUS, possivelmente pela perspectiva restritiva desta diretriz ao setor saúde.

Como contribuição, o grupo propôs a inclusão de duas diretrizes: promover experiências territorializadas de desenvolvimento sustentável e saúde e fortalecer o movimento de municípios/cidades saudáveis como parte da política pública do SUS.

\subsection{Temas prioritários da PNPS}

O grupo considerou como essencial a manutenção como temas prioritários na PNPS: prática corporal/atividade física; prevenção da violência e estímulo à cultura de paz; e promoção do desenvolvimento sustentável.

Por outro lado, apesar de terem sido considerados importantes, não houve consenso sobre a priorização dos temas mais específicos ligados a comportamentos de risco como o controle do tabagismo, uso abusivo de álcool e outras drogas e acidentes de trânsito.

O grupo de participantes apontou uma série de outros temas considerados importantes para serem priorizados pela PNPS entre eles: saúde mental; desenvolvimento de ambientes urbanos saudáveis; espiritualidade; respeito à diversidade; práticas integrativas e complementares; saúde social, convívio, lazer e cultura; cultura local e sustentabilidade; identificação de problemas e potencialidades nos territórios; promoção da saúde ambiental e da habitação saudável.

\subsection{Estratégias da PNPS}

Foram consideradas estratégias importantes para a implementação das ações de promoção da saúde estimuladas pela PNPS: a identificação de problemas nos territórios; a ampliação da eficiência e participação; o acesso a informação sobre recursos para promoção da saúde; bem como a capacitação de gestores de políticas públicas em promoção da saúde.

\subsection{Eixos operacionais da PNPS}

Houve consenso do grupo de especialistas quanto a considerar como essencial a manutenção dos eixos operacionais ligados a gestão e financiamento; participação e controle social; e ambientes promotores da saúde.

Apesar de terem sido considerados eixos importantes, com exceção de uma manifestação de pouca importância do eixo operacional ligado à comunicação e mídia, não houve consenso do grupo em relação à essencialidade de manutenção dos eixos operacionais ligados à vigilância e monitoramento, articulação intersetorial, regulação e controle, promoção da saúde nas redes de atenção à saúde, educação em saúde/autocuidado, comunicação e mídia e pesquisa e inovação.

Como sugestões, o grupo propôs a inclusão dos seguintes eixos operacionais: promoção de agendas territorializadas; diálogo com políticas sociais de equidade (movimentos sociais - diversidade sexual, população de rua, etc...); trabalho em rede no território; formação inicial de trabalhadores da saúde; capacitação em promoção da saúde e ação intersetorial dos diversos atores envolvidos (trabalhadores da saúde, gestores e trabalhadores ligados a outros setores); formulação de leis específicas para a construção de espaços urbanos saudáveis, além da sugestão de rever o eixo "Comunicação e mídia", ampliando para geração e difusão de conhecimento.

\subsection{Recomendações para a nova versão da PNPS}

Um dos respondentes destacou a necessária revisão do modelo estratificado por pautas (temas) na construção do texto da PNPS, o qual foi considerado não adequado para atender às demandas complexas de desenvolvimento de práticas de promoção da saúde. Esta observação foi reforçada pelo grupo, o qual apontou várias recomendações para oportunizar esta adequação da Política às atuais demandas de ações voltadas para a promoção da saúde, as quais estão listadas abaixo:

- Capacitação de gestores

- Política deveria ser interministerial

- Criação de Comitê Gestor Interministerial da PNPS, com participação dos movimentos sociais 
- Apoio a agendas territorializadas e redes voltadas para sua implementação

- Políticas indutoras que estimulem a cogestão

- Focalização e temas transversais

- Eventos e materiais de apoio para mobilização

- Divulgação e estímulo a boas práticas

- Comunicação permanente sobre promoção da saúde nos espaços sociais

- Fortalecimento/aceleração da implementação do Plano Nacional de Resíduos Sólidos e saneamento básico

- Aconselhamento da discussão da promoção da saúde na revisão dos Planos Diretores Municipais

- Capilarização e articulação intersetorial (Transporte, Meio Ambiente, Cultura, Educação, Esporte, Assistência Social, Ministério das Cidades, Artes, Instituições de Ensino Superior)

- Aproximação das ações de promoção da saúde com culturas populares, iniciativas comunitárias, organizações e movimentos sociais

- Fortalecimento do Programa Saúde na Escola, ampliando suas diretrizes

- Busca de parcerias para intervenções sustentáveis nos municípios

- Integração efetiva com setores que tratam do planejamento e gestão territorial

- Monitoramento e avaliação de efetividade da PNPS

\section{Considerações Finais}

A participação e responsabilidade de outros atores, não apenas de profissionais vinculados ao setor saúde, na formulação, implementação e avaliação da PNPS deve estar alicerçada em compromissos e conhecimentos produzidos no âmbito nacional, regional e local.

A busca da contribuição de especialistas vinculados a instituições fora do setor saúde e movimentos sociais envolvidos com ações intersetoriais de promoção da saúde oportunizou o fortalecimento da construção de uma nova versão da PNPS estabelecendo pontes para os diálogos intersetoriais que pretendem contribuir ainda mais para a necessária aproximação e suporte ao desenvolvimento destas ações no contexto brasileiro.
Referências

AKERMAN M.; FRANCO DE SÁ R.; MOYSES S.T.; REZENDE R.; ROCHA D. Intersetorialidade? IntersetorialidadeS!. Ciênc. saúde coletiva, v. 19, n. 11, p. 4291-4300, 2014.

BARDIN, L. Análise de conteúdo. Lisboa: Geográfica, 2009.

BRASIL. Lei no ${ }^{\circ}$ 8.080, de 19 de setembro de 1990. Dispõe sobre as condições para a promoção, proteção e recuperação da saúde, a organização e o funcionamento dos serviços correspondentes e dá outras providências. Diário Oficial da União, 19 set., 1990a.

BRASIL. Lei n ${ }^{\circ}$. 8.142, de 28 de dezembro de 1990. Dispõe sobre a participação da comunidade na gestão do Sistema Único de Saúde (SUS) e sobre as transferências intergovernamentais de recursos financeiros na área da saúde e dá outras providências. Diário Oficial da União; $31 \mathrm{dez}$, $1990 b$.

BRASIL. MINISTÉRIO DA SAÚDE. Política Nacional de Promoção da Saúde. Brasília: Ministério da Saúde; 2006.

BRASIL. MINISTÉRIO DA SAÚDE. Política Nacional de Promoção da Saúde: PNPS: revisão da Portaria MS/GM no 687, de 30 de março de 2006. Brasília: Ministério da Saúde; 2015.

BUENO, R. E.; MOYSES, S. T.; BUENO, P. A. R.; MOYSES, S. J. Governança, sustentabilidade e equidade no plano de saúde de São José dos Pinhais, Brasil. Rev Panam Salud Publica. v. 34, n. 6, p. 416-421, 2013.

COMMISSION ON SOCIAL DETERMINANTS OF HEALTH. A Conceptual Framework for Action on the Social Determinants of Health. Vancouver, 2007.

MALTA, D. C. SILVA, M. M. A.; ALBUQUERQUE, G. M.; LIMA, C. M.; CAVALCANTE, T.; JAIME, P. C.; SILVA JUNIOR, J. B. A implementação das prioridades da Política Nacional de Promoção da Saúde, um balanço, 2006 a 2014. Ciênc. saúde coletiva.v. 19, n. 11, p. 4301-4312, 2014.

MORETTI, A. C.; TEIXEIRA, F. F.; SUSS, F. M. B.; LAWDER, J. A. C.; LIMA, L. S. M.; BUENO, R. E.; MOYSÉS, S. J.; MOYSÉS, S. T. Intersetorialidade nas ações de promoção de saúde realizadas pelas equipes de saúde bucal de Curitiba (PR). Ciênc. saúde coletiva, v. 15, suppl. 1, p. 1827-1834, Jun. 2010.

ORGANIZAÇÃO MUNICIAL DA SAÚDE - OMS. Declaração de Adelaide sobre a Saúde em Todas 
as Políticas. Governo da Austrália Meridional, Adelaide, 2010.

ORGANIZAÇÃO MUNICIAL DA SAÚDE - OMS. Health in all policies (HiAP) framework for country action. Genebra: OMS; 2014. Disponível em:

<http://www.who.int/cardiovascular_diseases/14012 $\underline{\text { OHPRHiAPFramework.pdf?ua }=1>}$

PIOLA, S. F.; VIANNA, S. M.; VIVASCONSUELO, D. Estudo Delphi: atores sociais e tendências do sistema de saúde brasileiro. Cadernos de Saúde Pública, v. 18, supl., p. S181-S90, 2002.
SILVA, K. L.; SENA, R. R.; AKERMAN, M.; BELGA, S. M. M.; RODRIGUES, A. T. Intersetorialidade, determinantes socioambientais e promoção da saúde. Ciênc. saúde coletiva, v. 19, n. 11, p. 4361-4370, 2014.

THE HELSINKI STATEMENT ON HEALTH IN ALL POLICIES. The 8th Global Conference on Health Promotion. Helsinki, Finland, 2013.

YAÑEZ, G. R.; CUADRA, O. R. La técnica Delphi y la investigación en los servicios de salud. Ciencia y enfermeria, v.14, n. 1, p. 9-15, 2014. 